\title{
Ultrasonic cleaning of 3D printed objects and Cleaning Challenge Devices
}

\author{
Bram Verhaagen ${ }^{\mathrm{a}}$, Thijs Zanderink ${ }^{\mathrm{b}}$, David Fernandez Rivas ${ }^{\mathrm{a}, \mathrm{c}, *}$ \\ ${ }^{a}$ BuBclean, Institutenweg 25, 7521PH Enschede, The Netherlands \\ ${ }^{\mathrm{b}}$ Saxion, University of Applied Sciences, P.O. Box 70000, 7513 AB Enschede, The Netherlands \\ ${ }^{\mathrm{c}}$ Mesoscale Chemical Systems, University of Twente, Carre 1.339, P.O. Box 217, 7500 AE Enschede, The Netherlands
}

\section{A R T I C L E I N F O}

\section{Article history:}

Received 29 January 2015

Received in revised form 3 June 2015

Accepted 16 June 2015

Available online $\mathrm{xxxx}$

\section{Keywords:}

Additive manufacturing

3D printing

Bubble

Cleaning

Ultrasonic cleaning

Sonication

\begin{abstract}
A B S T R A C T
We report our experiences in the evaluation of ultrasonic cleaning processes of objects made with additive manufacturing techniques, specifically three-dimensional (3D) printers. These objects need to be cleaned of support material added during the printing process. The support material can be removed by dissolution in liquids with or without ultrasonic cavitation.

Distinctive stages in the cleaning processes were found for two different liquids (water and $\mathrm{NaOH}$ solutions). The combination of ultrasound and high concentration of $\mathrm{NaOH}$ has the best results for support material dissolution in the particular conditions studied.

The sonication of cleaning processes in ultrasonic baths is typically a slow process. Here we show the advantages of using an ultrasonic horn to clean the surface of small parts and holes more effectively.

We introduce a Cleaning Challenge Device design to be used for the universal evaluation of cleaning performance of different equipment or processes, and specifically for ultrasonic baths. The results and conclusions can be of use for different cleaning situations besides 3D printed parts, such as when deciding which protocol has a better performance or comparing different equipment.
\end{abstract}

(c) 2015 Elsevier Ltd. All rights reserved.

\section{Introduction}

Additive manufacturing techniques were invented several decades ago, but it was not until recently that three-dimensional (3D) printing has become popular and has pushed the imagination of users. The discovery of new functions and applications of relevance for many fields has been possible with the advent of 3D printing. Recently 3D printers have been used to fabricate spare parts for space missions, the printing of organs for transplantations, and novel structures and chemical reactors [1-4].

There are several different techniques for 3D printing, and also a diverse range of materials in use, each requiring dedicated post-processing procedures [5-7]. The post-processing can include polishing, brushing or painting the object; here we will refer to cleaning or removal of support material from the printed item. Support material is defined as the material that is added for stability during the printing process, and can either be a separate material or made out of the 3D printing material itself. When there are moving parts in an item, or small details that are crucial for the functioning of the object, the residues of support material or other contamination can render the object, or the whole item

* Corresponding author at: Mesoscale Chemical Systems, University of Twente, Carre 1.339, P.O. Box 217, 7500 AE Enschede, The Netherlands.

E-mail address: d.fernandezrivas@utwente.nl (D. Fernandez Rivas). non-functional, leading to undesired economical losses or health problems. The cleaning of printed parts can be complex and time consuming, taking up to $30 \%$ of the total production time, as expressed by companies that the authors talked with. Typically, the cleaning involves first manual removal of bulk support material (possibly assisted by a water jet), followed by ultrasound as a 'post-treatment'. These companies would like to spend less than $4 \mathrm{~h}$ on cleaning.

Ultrasonic baths have been widely used to clean objects for several decades [8-10]. Ultrasonic horns are known to create even stronger mechanical effects as it focuses the ultrasonic power into a small volume $[11,12]$. Therefore they make possible to clean the objects faster than with ultrasonic baths. It is known that the prolonged effect of bubbles collapsing close to surfaces can lead to erosion or destruction of the surface, depending on different factors, such as the type of cavitation: inertial or stable. For this reason it is important to ensure that the duration of the cleaning process, and the applied process parameters, such as the pressure amplitude and frequency, allow for certain degree of control over the desired cleaning while avoiding the damaging behavior [13-16].

The combination of materials and contamination that can be cleaned from a substrate is so varied that it is impossible to cover all in one single study. The properties of the substrate and contamination materials (hard-hard, soft-hard, soft-soft, acoustics), or geometrical shapes (flat substrates, tubes, intricate shape objects), 
as well as chemical compatibility with a cleaning solution, are among the different variables to be taken into account [17]. This investigation focuses on the ultrasonic cleaning (post-processing) of objects printed with UV-cured material deposition (Objet printers, Stratasys) [18]. The final object comes out of the 3D printer embedded in surrounding support material, which has different properties than the object itself. The support material is actually a heterogeneous combination of construction and support material; the support material part should be removed such that the remaining scaffold of construction material collapses. Near the walls, the support material mixture is different than the bulk support material - the so-called attachment layer. When the support material sits in geometrically complex objects, it becomes difficult to remove it, since the dissolving fluid must get in contact with these surfaces difficult to reach by hand or other methods. We provide a general protocol and evaluation methods to monitor the ultrasonic cleaning, by using two models that represent cleaning challenges.

Definitions of "what is clean", and methods to quantify cleaning processes, other than the subjective inspection by eye, are two aspects to consider before it is possible to automate such processes [17]. It is important to provide quantifiable variables that can be used to compare among the different cleaning steps, different cleaning equipment, and even the individuals performing the cleaning. In the semiconductor industry these questions have been answered to a certain extent [19]. An intuitive and simple procedure is to weight the sample before and after it is cleaned; the use of microbalances to monitor contaminant removal in ultrasonic cleaning applications has been reported [20], as well as micro-pores as model systems for particle removal from high aspect ratio structures [21]. In this article we outline several methods for quantitative cleaning measurement particularly oriented to ultrasound and 3D printed objects.

The cleaned state of a given object sometimes cannot be directly checked and there may be a risk of re-contaminating it. We borrow the concept of a process challenge device from the sterilization of medical tools, which typically consists of an object emulating the item with the most challenging feature to be cleaned. As an example, gke's Batch Monitoring System [22] is a hollow spiral with a closed end that is placed in the autoclave together with the medical instruments to be sterilized. After a sterilization process, the Batch Monitoring System allows to check if the conditions for sterilizing inside the autoclave were reached, without compromising the sterility of the real instruments. Up to now we found no reference in literature of a Cleaning Challenge Device (CCD) that could be used for monitoring the efficiency of an ultrasonic cleaning method, or comparing one post-processing technique with another. We present our proposition of a CCD for the ultrasonic cleaning of 3D printed parts, which could also be used to evaluate other post-processing techniques, or to compare the efficiency of different cleaning methods.

\section{Materials and methods}

\subsection{Sample fabrication and characterization}

All the samples were fabricated with an Objet Eden 350V UV-curing material deposition 3D printer. In a pilot study, five pieces of approx. $10 \times 10 \times 8 \mathrm{~mm}$ were cut from support material weighing $1.00 \pm 0.01 \mathrm{~g}$, and placed in ultrasonic cleaning bags (BuBble bags, BuBclean) [23].

- Sample 1, with $10 \mathrm{~mL}$ of demi-water.

- Sample 2, with $1.5 \mathrm{M} \mathrm{NaOH}(0.6 \mathrm{~g} \mathrm{NaOH}$ dissolved in $10 \mathrm{~mL}$ of demi-water).

- Sample 3, with $1.5 \mathrm{M} \mathrm{NaOH}$, and was kept at $30^{\circ} \mathrm{C}$.

- Sample 4 , with $10 \mathrm{~mL}$ of demi-water, placed in an ultrasonic bath (at $30^{\circ} \mathrm{C}$ ) for $2 \times 15$ min with $45 \mathrm{~min}$ in-between.

- Sample 5, with $1.5 \mathrm{M} \mathrm{NaOH}$ and was also placed in an ultrasonic bath $\left(\right.$ at $30^{\circ} \mathrm{C}$ ) for $2 \times 15 \mathrm{~min}$ with $45 \mathrm{~min}$ in-between.

In total, each sample resided in the plastic bag for $2 \mathrm{~h}$ and was then left to dry for $1 \mathrm{~h}$. Afterward, the samples were weighed again. This allowed finding the optimal conditions for the experiments in the remaining studies presented here. Following this pilot study, a more detailed experiment was set up in which the weight change after 10 min intervals was measured. The concentration of $\mathrm{NaOH}$ was varied from $0.5 \mathrm{M}$ to $6 \mathrm{M}$.

For the cleaning of 3D printed objects two Cleaning Challenge Models were used. The first type $(A)$ were extruded squares of $10 \times 10 \times 3 \mathrm{~mm}$, attached to an extruded polygon for handling purposes (Fig. 1A). The second model (B) had two variations. Model B. 1 was a block of dimensions $65 \times 25 \times 130 \mathrm{~mm}$ with two closed-end channels of diameter 5 and $1 \mathrm{~mm}$ and $100 \mathrm{~mm}$ length (Fig. 1B). Model B.2 was designed with channels of 2.5, 1 and $0.5 \mathrm{~mm}$ diameter, and $80 \mathrm{~mm}$ long holes. At the top of both model B blocks, a small chamber of $61 \times 21 \times 31 \mathrm{~mm}$ allowed liquid containment. The chamber and the channels were filled with support material during the printing process. Models A and B were printed in VeroWhitePlus material (Stratasys), an acrylic compound.

The bulk support material around the printed objects was removed by hand, leaving only small remnants and a very thin (connecting) layer of support material. Finally, loose remnants were removed by low-flux flushing with water.

Infrared absorption measurements provided an indication of the chemical groups present in the support material samples. An
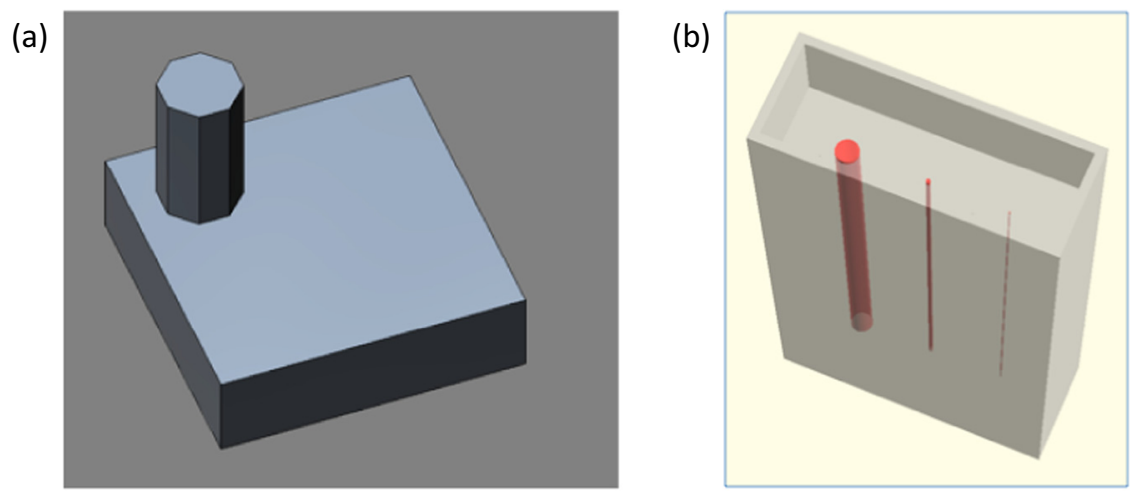

Fig. 1. (a) Test model with dimensions of the square block $10 \times 10 \times 3 \mathrm{~mm}$ and (b) block with close-end channels with total dimensions of $65 \times 25 \times 130 \mathrm{~mm}$. 
infrared FT-IR Tensor (FT-IR Tensor27, Bruker) was used for these experiments. A High Resolution Scanning Electron Microscope (SEM; 1550, Zeiss) was used to visualize in detail the remaining support material on ultrasonically cleaned printed objects. A magnification of $100-1000 \times$ was used, with a voltage of $4 \mathrm{kV}$. Images were taken with a photo-camera (D900, Nikon) and macro-lens (50 mm, Sigma). A balance (APX-100, Denver Instrument) was used to weight the samples before and after each experiment, with an error margin of $0.1 \mathrm{mg}$.

\subsection{Ultrasonic equipment and cleaning chemical solutions}

An ultrasonic bath (USC200TH, VWR) operating at $45 \mathrm{kHz}$ was used for the dissolution of support material experiments. The samples were placed in the ultrasonic bath and contained inside a $200 \mathrm{~mL}$ beaker supported by the lid of the bath. An ultrasonic horn (Sonopuls HD3200, Bandelin), with a tip of $2 \mathrm{~mm}$ diameter (MS72, Bandelin), was used to clean the printed models. This device was operated at $30 \%$ of its maximum power $(200 \mathrm{~W})$, and with duty cycles of $50 \%$ (i.e. pulses of $0.5 \mathrm{~s}$ in every $1 \mathrm{~s}$ ) to avoid excessive heating and permanent deformation of the printed object. Stratasys advises to use a $0.5 \mathrm{M} \mathrm{NaOH}$ solution for the removal of support material and cleaning the printed parts [24]. A $2 \% \mathrm{NaOH}$ $(0.5 \mathrm{M})$ solution was prepared by dissolving $0.99 \mathrm{~g}$ of $\mathrm{NaOH}$ (Sigma Aldrich) in $50 \mathrm{~mL}$ of miliQ water. A $6 \mathrm{M} \mathrm{NaOH}$ solution was prepared by dissolving $11.99 \mathrm{~g}$ of $\mathrm{NaOH}$ in $50 \mathrm{~mL}$ of purified water, to study the effects of increased concentration.

\section{Results}

\subsection{Dissolution of support material}

The samples submerged in water alone at room temperature showed no signs of dissolving. Their final weight had increased from $1.00 \pm 0.01 \mathrm{~g}$ to $1.13 \pm 0.01 \mathrm{~g}$, which suggests that they had absorbed water. All samples submerged in $\mathrm{NaOH}$ showed a reduction both in size and in weight, even though they also may have absorbed liquid. In $\mathrm{NaOH}$ at room temperature, the final weight was $0.58 \mathrm{~g}$; at $30^{\circ} \mathrm{C}$, the final weight was $0.47 \mathrm{~g}$. When ultrasound was also present, the final weight was $0.32 \mathrm{~g}$. Each sample can be seen in Fig. 2.

Fig. 3 shows a typical graph of the sample weight percentage change $\left(W_{\text {time }} / W_{\text {initial }} \times 100 \%\right)$ measured every $10 \mathrm{~min}$. We term the first 60 min as the active cleaning phase, in which the object is cleaned by ultrasound or another technique. In the first part of this period the weight can increase due to swelling of the sample by absorbing water. Support material is removed in this phase, but the weight loss is masked by the swelling effect. As the support material is dissolved or mechanically removed, the weight decreases up to the Post-Cleaning value, $\mathrm{PC}_{\mathrm{a}}$. The passive cleaning phase is the period from 60 to $1200 \mathrm{~min}$, where the sample is left in a room conditions environment $\left(25^{\circ} \mathrm{C}, 1 \mathrm{~atm}\right)$ allowing the excess fluid to evaporate, and is weighted once more $\left(\mathrm{PC}_{\mathrm{b}}\right)$.

Each experimental setting was repeated three times, and the weight values were averaged. The results of the percentage weight change for all three cases studied are shown in Fig. 4.

In water the ultrasound effect resulted in a final weight loss $20 \%$ larger than in silent conditions. For $0.5 \mathrm{M} \mathrm{NaOH}$, the ultrasound increased the final weight loss with roughly $10 \%$ compared to silent conditions. The $6 \mathrm{M} \mathrm{NaOH}$ shows a final weight loss of roughly more than $20 \%$ bigger than in silent conditions. The percentage change values after the post-cleaning active $\left(\mathrm{PC}_{\mathrm{a}}\right)$ and passive $\left(\mathrm{PC}_{\mathrm{b}}\right)$ phases of all experiments are shown in Table 1. From these values we observe that the case of ultrasound always gives the best results, and particularly the higher concentration of $\mathrm{NaOH}$ removes more material.

The active cleaning rate ( $\left.d_{\text {active }}\right)$, passive $\left(d_{\text {passive }}\right)$ and, average $\left(d_{\text {average }}\right)$ were calculated in $\mathrm{g} / \mathrm{min}$ and are derived from the formulas:

$$
\begin{aligned}
& d_{\text {active }}=\Delta W_{\text {active }} / T_{\text {active }} \\
& d_{\text {passive }}=\Delta W_{\text {passive }} / T_{\text {passive }} \\
& d_{\text {average }}=\left(d_{\text {active }}+d_{\text {passive }}\right) / 2
\end{aligned}
$$

where $\Delta W$ is the difference in weight and $T_{\text {active }}$ and $T_{\text {passive }}$ are the time of active or passive cleaning respectively. These values are used to determine the best cleaning method for a generic object

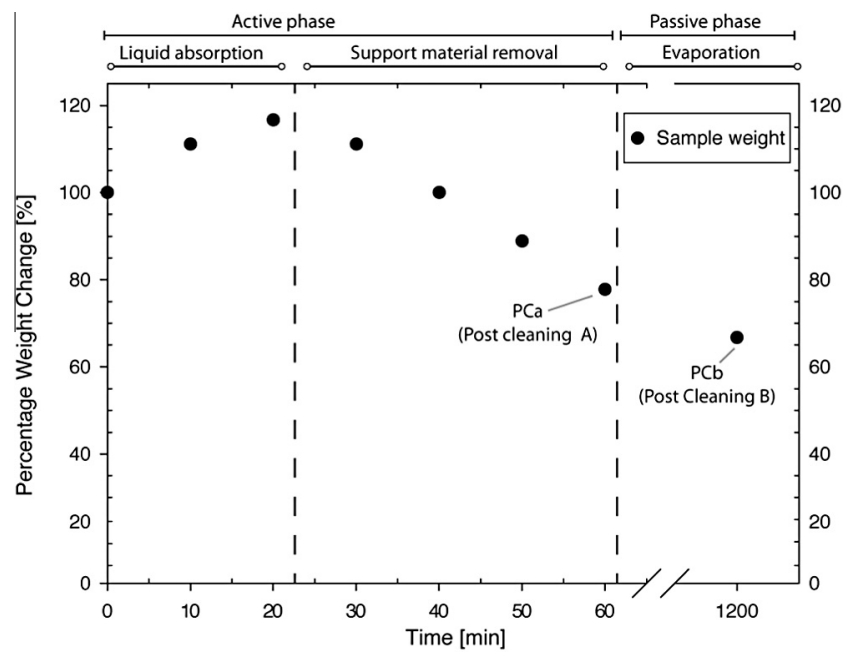

Fig. 3. Typical graph illustrating the two experiment phases of the percentage weight change: active (first $60 \mathrm{~min}$ ) and passive (60-1200 $\mathrm{min}$ ). The first part of the passive phase can show weight increase due to swelling. The $P_{a}$ and $P C_{b}$ values are the final weights of each phase.

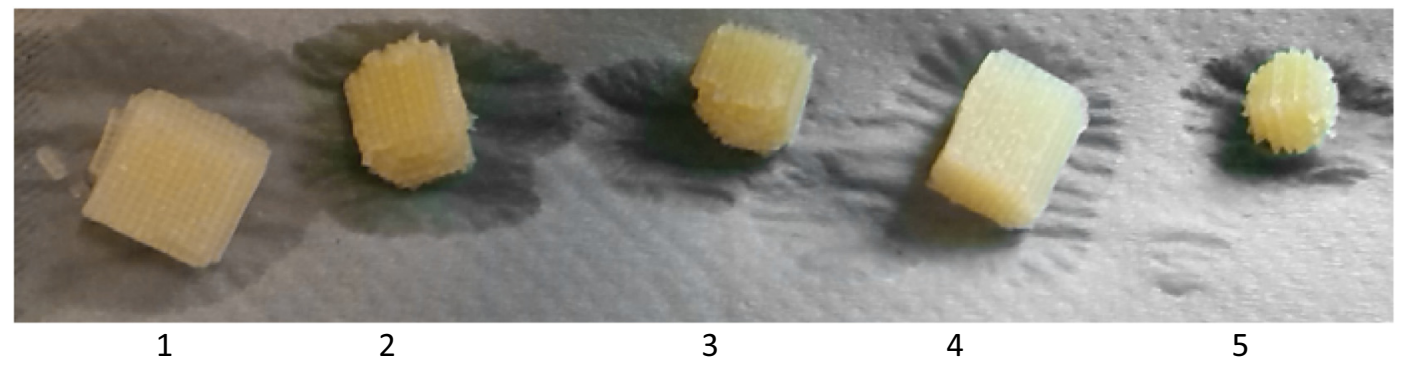

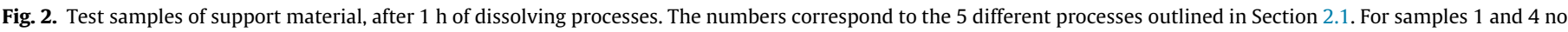
dissolution was visible, i.e. their shape is identical to the original samples $(10 \times 10 \times 8 \mathrm{~mm})$. 

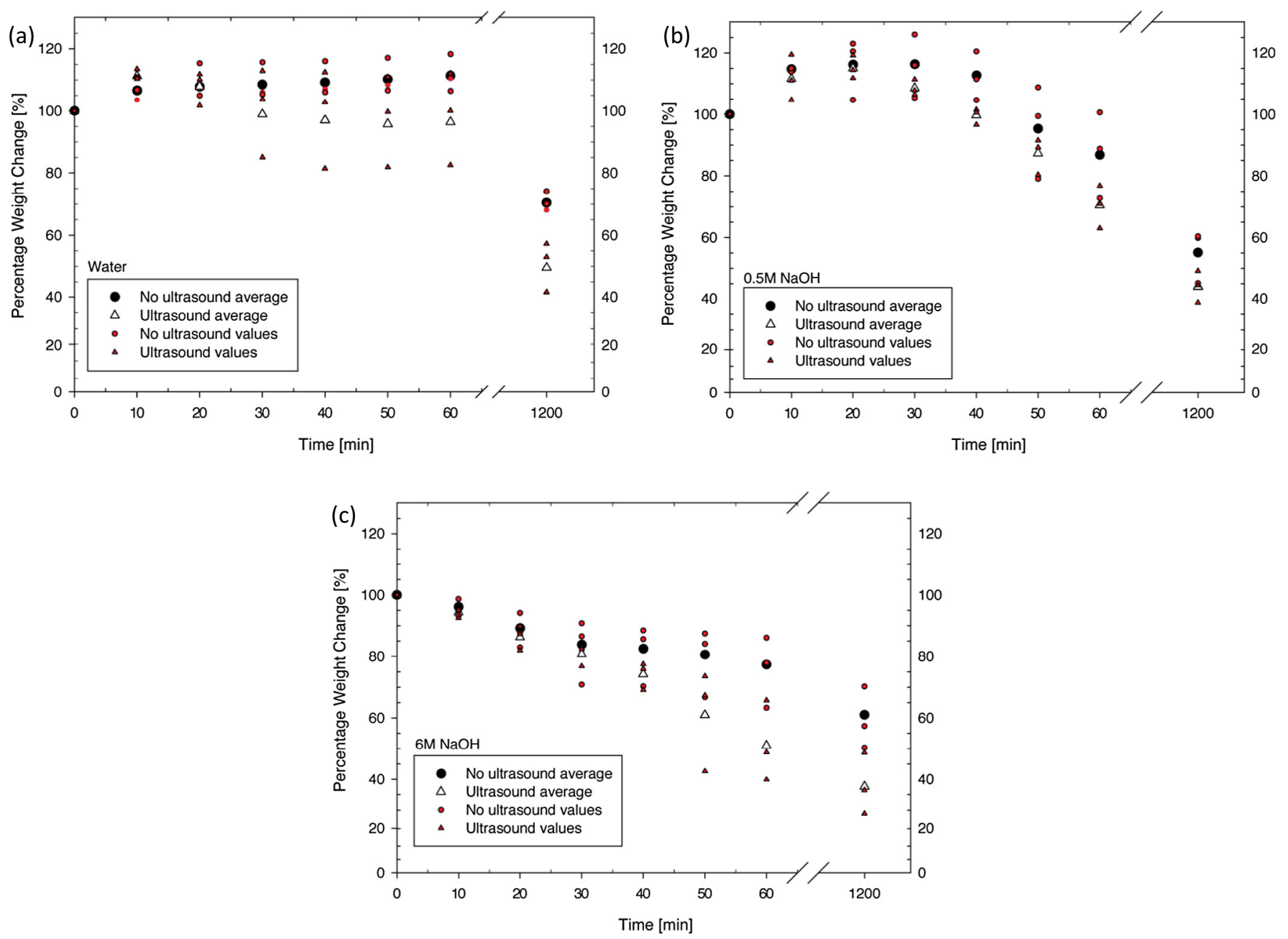

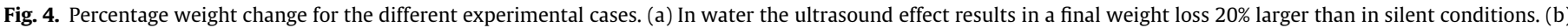

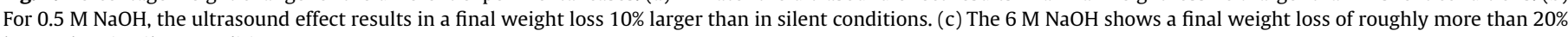
larger than in silent conditions.

Table 1

Percentage change values for the experimental conditions studied at post-cleaning points $\mathrm{PC}_{\mathrm{a}}$ and $\mathrm{PC}_{\mathrm{b}}$.

\begin{tabular}{|c|c|c|c|c|c|c|}
\hline \multirow{2}{*}{$\begin{array}{l}\text { Solution time } \\
\text { (min) }\end{array}$} & \multicolumn{3}{|c|}{ No ultrasound } & \multicolumn{3}{|c|}{ Ultrasound } \\
\hline & $\mathrm{H}_{2} \mathrm{O}$ & $\begin{array}{l}0.5 \mathrm{M} \\
\mathrm{NaOH}\end{array}$ & $\begin{array}{l}6 \mathrm{M} \\
\mathrm{NaOH}\end{array}$ & $\mathrm{H}_{2} \mathrm{O}$ & $\begin{array}{l}0.5 \mathrm{M} \\
\mathrm{NaOH}\end{array}$ & $\begin{array}{l}6 \mathrm{M} \\
\mathrm{NaOH}\end{array}$ \\
\hline $\mathrm{PC}_{\mathrm{a}}$ (after $1 \mathrm{~h}$ ) & 111.32 & 86.85 & 77.33 & 96.41 & 70.46 & 50.99 \\
\hline $\mathrm{PC}_{\mathrm{b}}($ after $20 \mathrm{~h})$ & 70.42 & 55.15 & 60.97 & 49.59 & 44.26 & 37.65 \\
\hline
\end{tabular}

or support material. The calculated cleaning rate values given in Eqs. (1)-(3) ( $d_{\text {active }}, d_{\text {passive }}$ and $\left.d_{\text {average }}\right)$ are plotted in Fig. 5. The highest dissolution rate is achieved with $0.5 \mathrm{M} \mathrm{NaOH}$ when using ultrasound, which has comparable values to the $6 \mathrm{M} \mathrm{NaOH}$, that shows the best removal values for the type of material and ultrasonic bath used in this study.

\subsection{Infrared spectroscopy}

The support material surface composition was analyzed with infrared spectroscopy, and the results are shown in Fig. 6. The region near $3500 \mathrm{~cm}^{-1}$ (marked 1) indicates the presence of $-\mathrm{OH}$ or $\mathrm{N}-\mathrm{H}$ bonds. The peak at $1550 \mathrm{~cm}^{-1}$ (2) corresponds to $\mathrm{C}=\mathrm{O}$ or $\mathrm{N}-\mathrm{H}$ bonds. The peaks around $1000 \mathrm{~cm}^{-1}$ (3) are attributed to

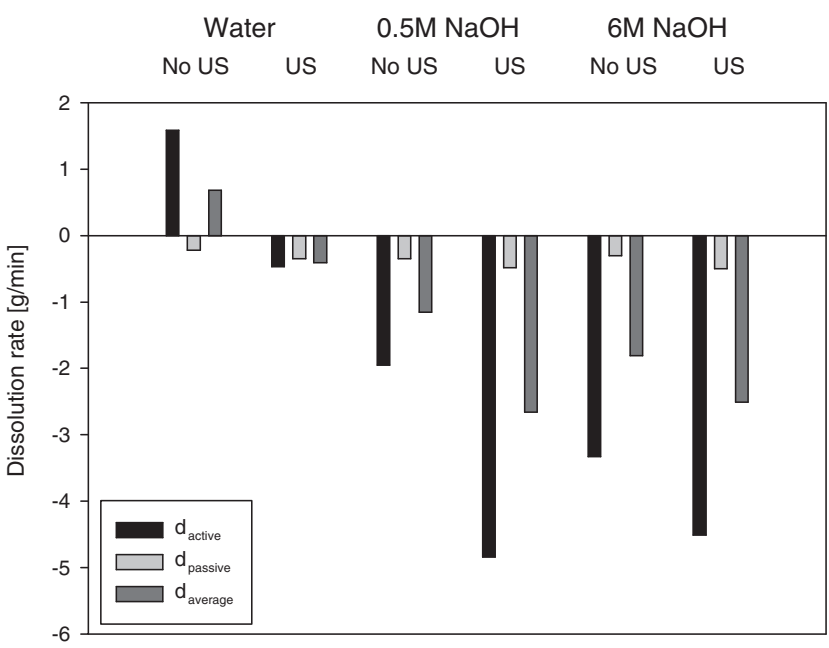

Fig. 5. Dissolution rate values as calculated with Eqs. (1)-(3). Only in the case of water without ultrasound an increase in weight is seen due to swelling by water absorption. The $0.5 \mathrm{M}$ and $6 \mathrm{M} \mathrm{NaOH}$ solutions show comparable $d_{\text {active. }}$.

$\mathrm{C}-\mathrm{O}$ or $\mathrm{C}-\mathrm{N}$ bonds. These results indicate that the sample contains a compound in ester form, as stated in the material ingredient database in which, besides the acrylic monomer $(<30 \%)$, it has 


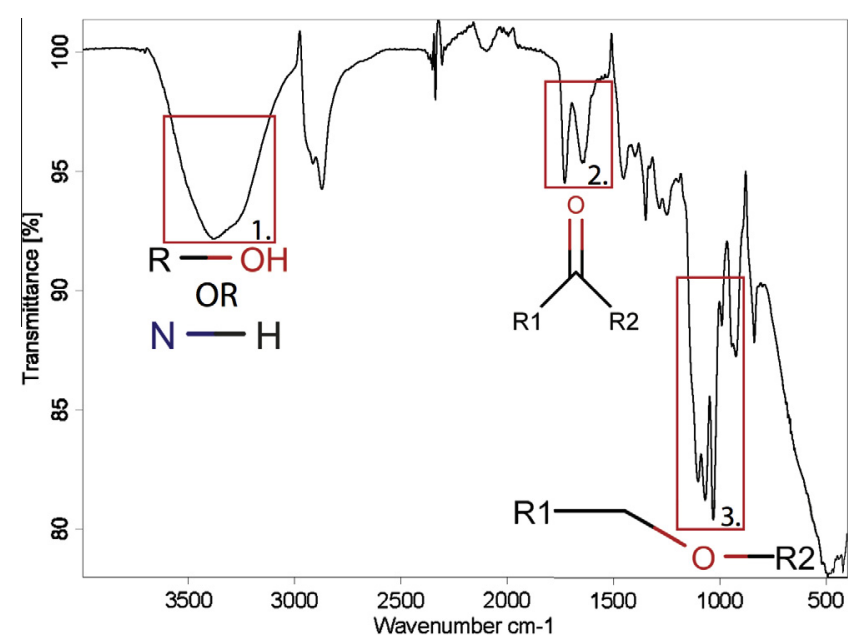

Fig. 6. Transmittance values of infrared measurements with distinctive features: (1) the region near $3500 \mathrm{~cm}^{-1}$ indicate the presence of $-\mathrm{OH}$ or $\mathrm{N}-\mathrm{H}$ groups. (2) The peak around $1550 \mathrm{~cm}^{-1}$ corresponds to $\mathrm{C}=\mathrm{O}$ or $\mathrm{N}-\mathrm{H}$ bonds. (3) The peaks around $1000 \mathrm{~cm}^{-1}$ are attributed to $\mathrm{C}-\mathrm{O}$ or $\mathrm{C}-\mathrm{N}$ groups.

acrylic acid ester $(<0.3 \%)$ [25]. These compounds when reacting with water form an alcohol and an acid.

No change in the in the apparent material composition was observed visually. More detailed studies can be carried out in future work to support this observation.

\subsection{Evaluation of cleaning printed objects}

The printer used in this study adds distinct support material as the print material is deposited, which can largely but not completely be removed manually. Especially challenging are sharp corners, small features and internal spaces. Different techniques were used to analyze the surface of the objects and identify the support and print material, hence providing an indication of "cleanliness".

\subsubsection{Microscopy}

SEM images allowed for observing the layered structure of the printed models. The support material can be recognized as it shows a different texture than the construction material (Fig. 7).

A disadvantage of SEM imaging is that it is time consuming and offers a limited field of view, which is not practical for the typical sample sizes used in 3D printing activity. For this reason we also performed optical microscopy (BX-FM, Olympus) as shown in Fig. 8, which shows remnants of support material as observed under $10 \times$ magnification.

\subsubsection{Image processing}

Macroscopically, the support material can be recognized as areas of light reflection, since the support material reflects light better than the construction material. We built a setup for photographing 3D printed objects, and perform image-processing analysis (color change, contrast enhancement, frequency filters), such that cleaning can be quantified. Figs. 9 and 10 show images used for the quantification process.

\subsection{Ultrasonic cleaning}

When operating standard ultrasonic baths, such as used in Section 3.1, the cleaning time can last longer than if a high-power device such as an ultrasonic horn is used. For this reason, the ultrasonic horn tip was positioned within $1 \mathrm{~mm}$ of the Cleaning Challenge Models and moved in steps across the surface. Ultrasound was applied at each position for a few seconds. Photographs of the surface were taken after each line across the surface to evaluate the removal process.
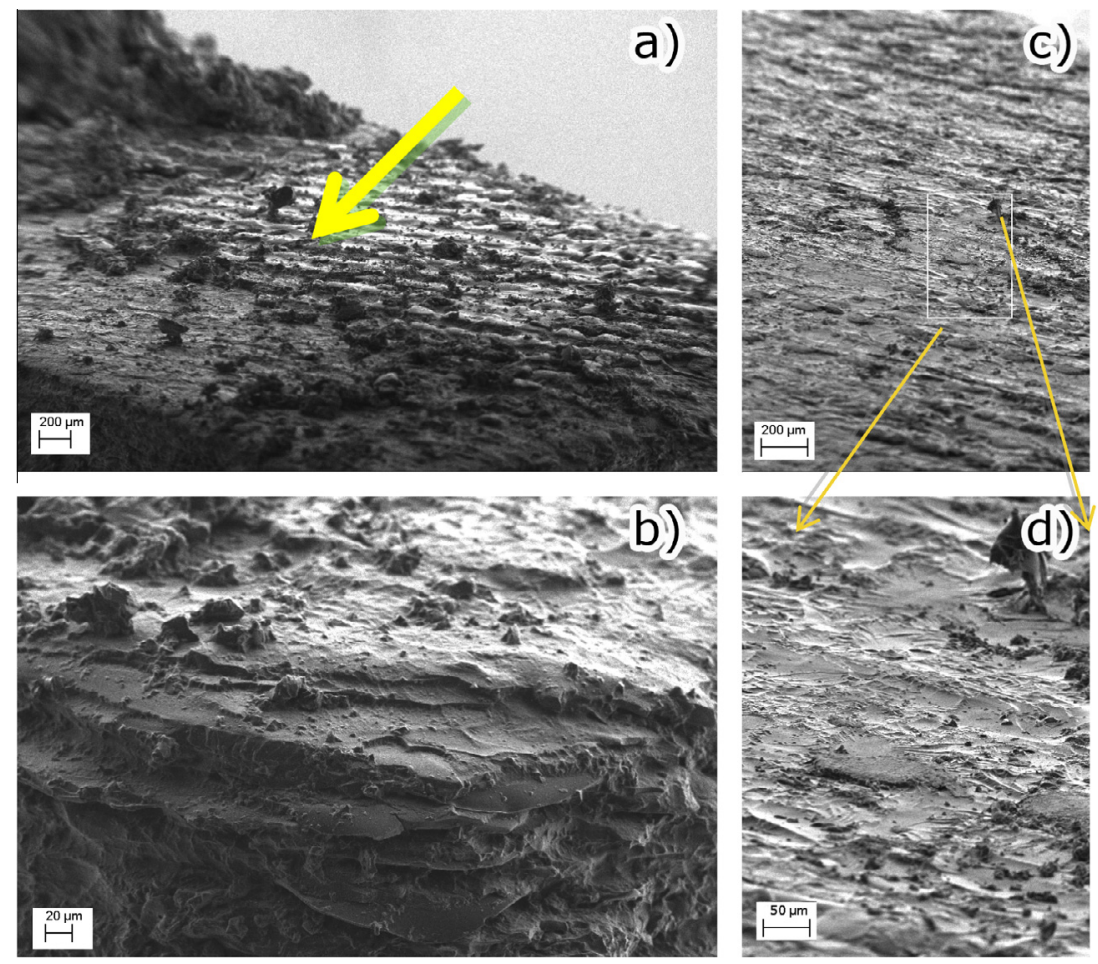

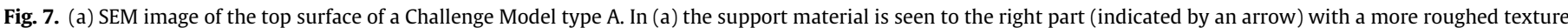

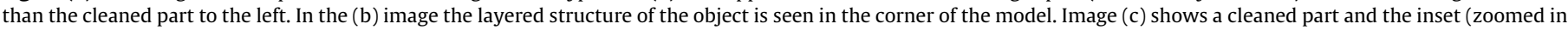
d) shows the smoother surface of the print material that was visually clean. 


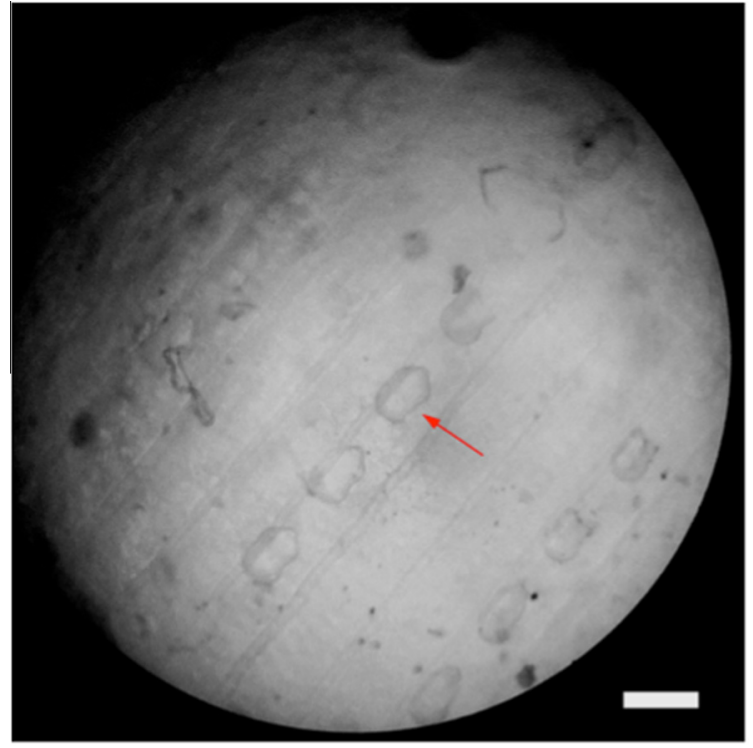

Fig. 8. $10 \times$ magnification of remaining support material after manual removal. The red arrow indicates a piece of support material. The white bar represents $50 \mu \mathrm{m}$. (For interpretation of the references to color in this figure legend, the reader is referred to the web version of this article.)

\subsubsection{Cleaning Challenge Model A}

In Fig. 11, the top surface of a model A shows remnant support material (the attachment layer) as more light reflecting areas.

The total time to achieve $100 \%$ support material removal was less than $5 \mathrm{~min}$. The rate of cleaning was calculated from the tip exposure area $\left(7 \mathrm{~mm}^{2}\right)$, achieved within one ultrasound pulse (i.e. one second). The cleaning rate was therefore $7 \mathrm{~mm}^{2} / \mathrm{s}$. The duty cycle and tip movement speed and pattern can be varied to optimize this process.

\subsubsection{Cleaning Challenge Model B}

The majority of the bulk material in Model B was first removed by hand as shown in Fig. 12. Each model was placed inside a $5 \mathrm{~L}$ glass beaker filled with tap water or a $0.5 \mathrm{M} \mathrm{NaOH}$ solution, at

(a)

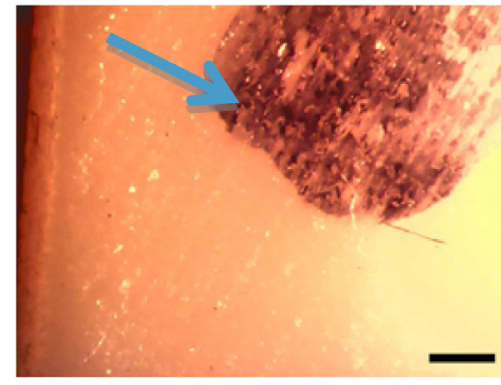

(c)

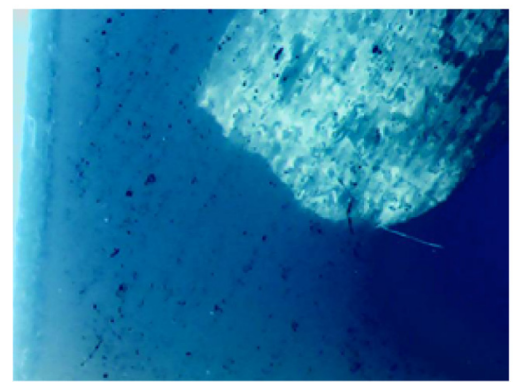

room temperature. The ultrasonic horn was used for cleaning the holes inside of the model, while the model was moved manually underneath the horn tip. In the Model B.1, the channels with diameters of 5 and $1 \mathrm{~mm}$ were cleaned with the ultrasonic power setting at $70 \%$, using only demi-water.

Fig. 13a shows the progression of the cleaned depth as a function of time. The first $20 \mathrm{~mm}$ of the $5 \mathrm{~mm}$-channel was cleaned 'remotely', i.e. by placing the ultrasonic horn tip just above the entrance of the channel. The removal rate was $2.8 \mathrm{~mm} / \mathrm{min}$. Beyond $20 \mathrm{~mm}$, the ultrasonic horn tip was allowed to make contact with the support material, and the cleaning rate increased to $7.5 \mathrm{~mm} / \mathrm{min}$. At $49 \mathrm{~mm}$ depth, the tapered horn tip binds in the channel (the place where the tip cannot be further pushed inside the channel); the removal rate beyond this point was $2.5 \mathrm{~mm} / \mathrm{min}$. The $5 \mathrm{~mm}$ channel was cleaned to a final depth of $69 \mathrm{~mm}$. This was $20 \mathrm{~mm}$ further than the point where the horn tip bound in the channel.

The cleaned depth as a function of time in the case of Model B.2 is presented in Fig. 13b. This model was treated by the ultrasonic horn at $50 \%$ power. Two copies of this model were printed; one was cleaned using demi-water and the other using $0.5 \mathrm{M} \mathrm{NaOH}$ solution. The cleaning rate for the $2.5 \mathrm{~mm}$ channel was $2.9 \mathrm{~mm} / \mathrm{min}$ with water, and $3.3 \mathrm{~mm} / \mathrm{min}$ with $\mathrm{NaOH}$. The final cleaned depth was $3.5 \mathrm{~mm}$ deeper with $\mathrm{NaOH}$ than with water. The cleaning rate for the $1 \mathrm{~mm}$ channel was $2.4 \mathrm{~mm} / \mathrm{min}$, both for water and $\mathrm{NaOH}$. With water, the removal process stopped at $6 \mathrm{~mm}$, whereas with $\mathrm{NaOH}$ the removal continued during the studied time. Note that the removal rate was initially approximately the same as for the $1 \mathrm{~mm}$ channel in model B.1, but since the ultrasound power is lower (50\% versus $70 \%$ ), the final depth is only $6 \mathrm{~mm}$. The cleaning rate for the $0.5 \mathrm{~mm}$ channel was $1.1 \mathrm{~mm} / \mathrm{min}$; the final depth was $4.5 \mathrm{~mm}$ (with water) or $6.7 \mathrm{~mm}$ (with $\mathrm{NaOH}$ ).

\section{Discussion}

\subsection{Support material dissolution}

From the initial experiment of support material dissolution it could be seen that the combination of ultrasound and $\mathrm{NaOH}$ had

(b)

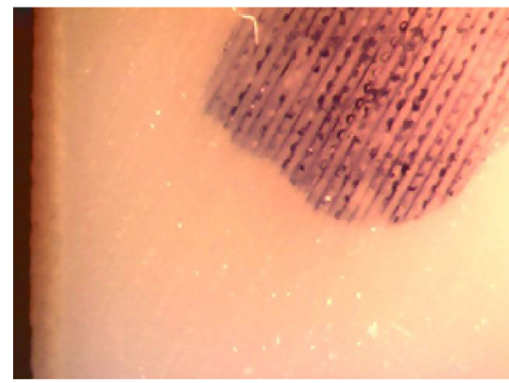

(d)

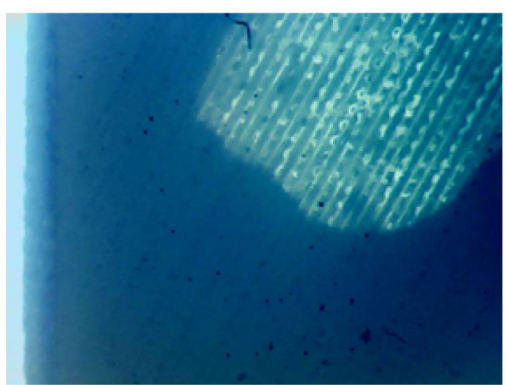

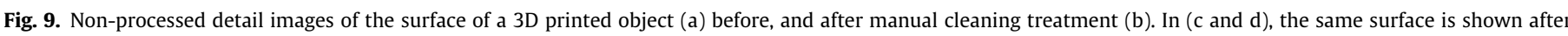

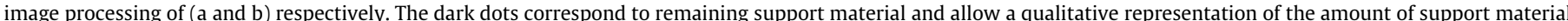

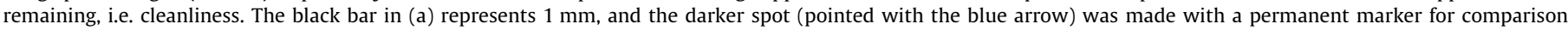
purposes. (For interpretation of the references to color in this figure legend, the reader is referred to the web version of this article.) 
(a)

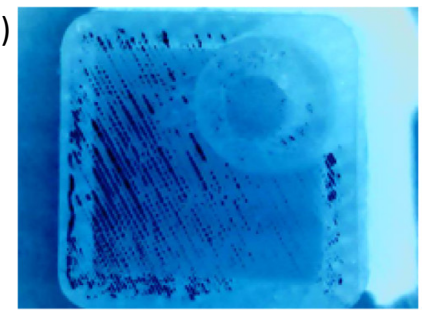

(b)

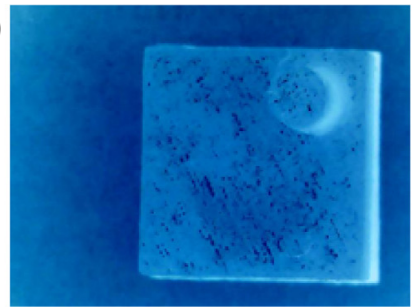

(c)

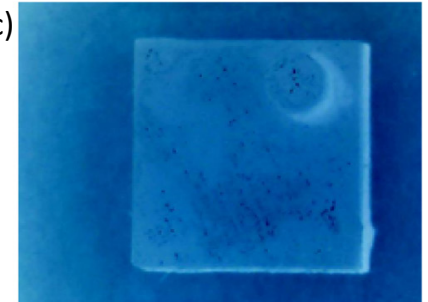

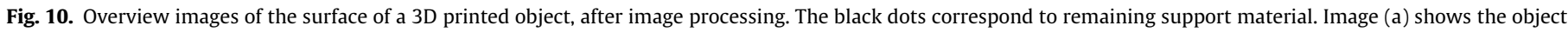

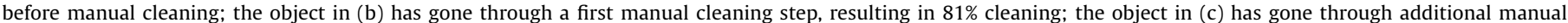
rubbing and was 95\% cleaned; see Fig. 1 for dimensions.

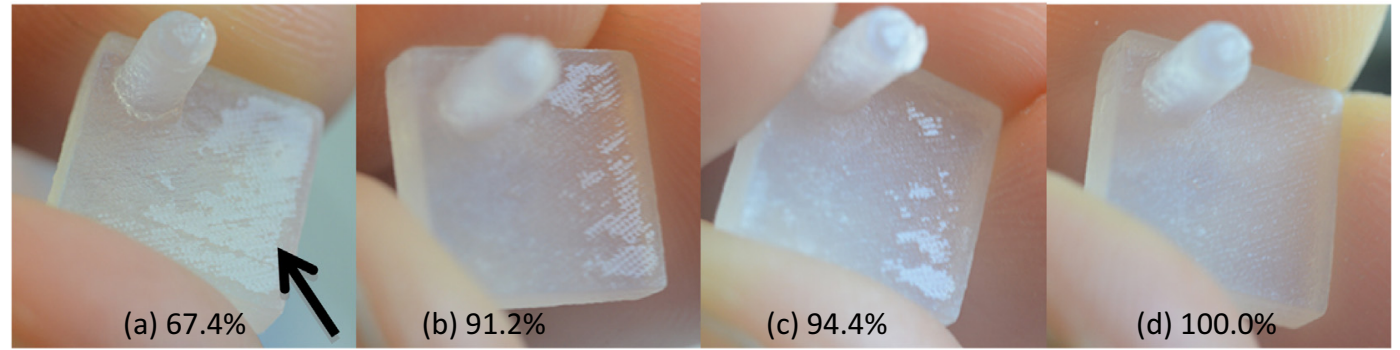

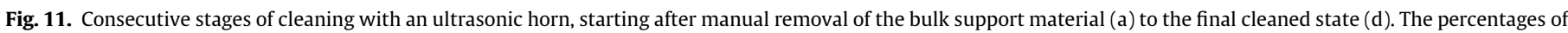
the cleaned surface is indicated, the arrow points at support material; see Fig. 1 for dimensions.

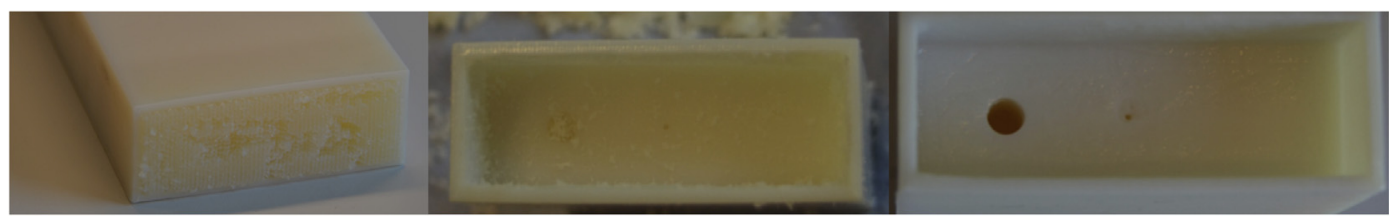

(a)

(b)

(c)

Fig. 12. Bulk support material in the chamber of Model B.1, before (a) and after (b) manual removal and (c) ultrasonic horn removal.

the best cleaning effect (see Fig. 4). NaOH is more effective in dissolving the support material than water. The material provider advises the use of $\mathrm{NaOH}$ solutions as cleaning medium, though acidic or surfactant solutions could be employed as well. In selecting other cleaning solutions, one should take into account the negative effects it might have on the polymer used to print the $3 \mathrm{D}$ objects.

The effect of heating is also demonstrated, since the amount of dissolved support material at $30{ }^{\circ} \mathrm{C}$ increased from $42 \%$ to $53 \%$ compared to room temperature (not taking into account any absorbed liquid). Agitation by ultrasound yielded the most dissolved support material: $68 \%$ after $2 \mathrm{~h}$. The rate of dissolution was roughly $0.25 \mathrm{~g} / \mathrm{h}$. This rate will be strongly dependent on the surface area of each printed object, and may be enhanced at other concentrations of $\mathrm{NaOH}$ or temperature, or more effective ultrasonic agitation.

Ultrasonic agitation will be most effective when the ultrasonic bath is used optimally, i.e. when parameters such as the temperature, gas content, filling height, ultrasound frequency and pulsing as well as sample positioning, are chosen correctly [26]. The effect of ultrasound leads to an increase of weight loss of $20 \%$ compared to the samples left in water without any agitation in one hour, while in our pilot experiments it was even $68 \%$ difference after $2 \mathrm{~h}$. In the case of only water (Fig. $4 \mathrm{a}$ ), for both the silent and US conditions the curve is flat for most of the experiment. The weight increase in the "active phase" of non-sonicated samples comes from swelling due to liquid absorbed in the material. The dissolution of the support material in the ultrasonic treated samples is increased by $20 \%$. The graphs for $6 \mathrm{M} \mathrm{NaOH}$ show a change in the curve shape during the first $30 \mathrm{~min}$, with an apparent decrease in swelling when compared with $0.5 \mathrm{M} \mathrm{NaOH}$ and water. When using $\mathrm{NaOH}$ solution of different concentrations the shape of the curve changes as can be seen in Fig. $4 \mathrm{~b}$ and c, with the more concentrated solution (6 M) showing a steeper slope of dissolution rate.

Among the sonication effects that can speed up the removal of support material we can mention acoustic streaming, bubble cavitation effects such as jetting and shockwaves, and chemical reactions as a result of radical generation. Radicals can degrade the polymers present in the printed and support material, and undergo base-hydrolysis to generate acrylic acid and acrylamide. The acrylic acid ester present in the support material (Fig. 6, Section 3.2) can react with water (hydrolysis) and form an acid and an alcohol, both soluble in water [27]. When a base solution (such as $\mathrm{NaOH}$ ) is added, saponification will occur, which results in an alcohol, a sodium ion, and a conjugated base of the acid forming the ester. If the solution $\mathrm{pH}$ is lowered at the final stage of a saponification reaction, the reversibility of the reaction is reduced, which could be beneficial for removing the support material permanently. In our case, since the concentration of $\mathrm{NaOH}$ used is high, the small decrease in $\mathrm{pH}$ that can occur due to nitric acid produced in the $\mathrm{N}_{2}+\mathrm{O}_{2}$ reaction will be negligible.

It is known that at low ultrasonic frequencies such as used in this study $(45 \mathrm{kHz})$ the mechanical effects of ultrasound (jets, streaming and shockwaves) are more significant than radical production; yet, since the support material might be composed of poly-ester, the radical generation as a result of ultrasonic 

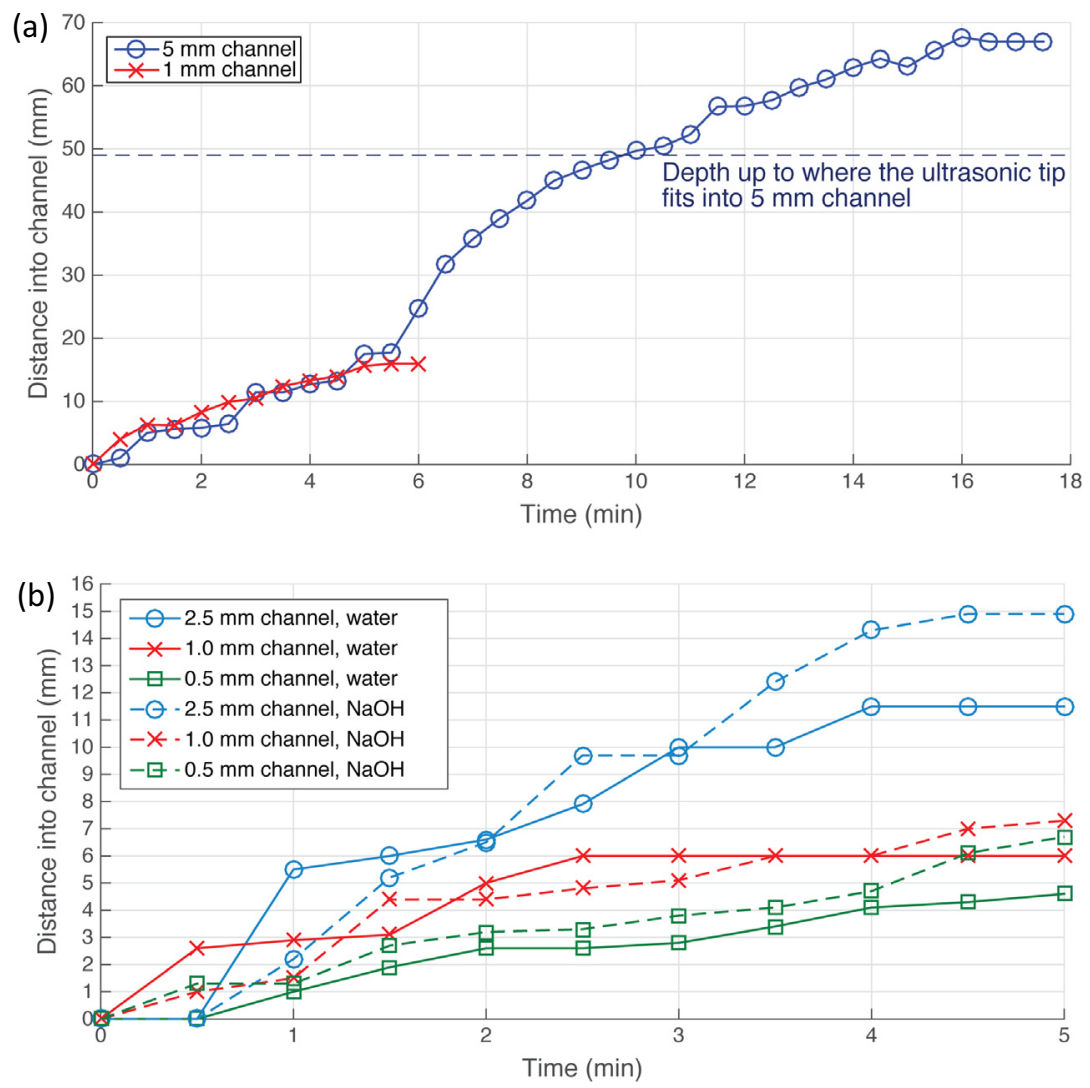

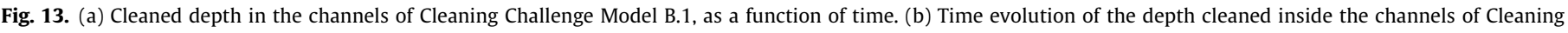
Challenge Model B.2 for water and $0.5 \mathrm{M} \mathrm{NaOH}$ solution.

cavitation could contribute to the hydrolysis reactions $[13,28]$. We speculate that the radical generation $\left(\mathrm{H}^{\cdot}\right.$ and $\left.\mathrm{OH}^{\circ}\right)$ known to occur in ultrasonic cavitation could contribute to an accelerated dissolution of the support material. Both radicals can attack the ester group at different bonds, accelerating the reaction. Further studies are needed to quantify this effect $[29,30]$.

In practical situations a protocol that uses the least amount of chemicals and energy consumption is always desirable. However, the higher concentration of $\mathrm{NaOH}$, even though it has a better removal effect, has a lower $\mathrm{pH}$ that can result in negative effects such as material corrosion. Handling such basic solutions increases the complexity of the cleaning processes, and has a non-negligible environmental impact when disposing the remaining solution after treatment. Additionally, the $0.5 \mathrm{M} \mathrm{NaOH}$ solution showed a higher active dissolution rate, which might be useful for processes where a shorter post-processing time is required, such as with fragile structures. Even though we did not look at the traces of $\mathrm{NaOH}$ in the cleaned surfaces, an additional cleaning step to neutralise $\mathrm{NaOH}$ should be performed to ensure the durability of the printed object.

\subsection{Ultrasonic cleaning of $3 D$ printed models}

In Section 3.4.1 we demonstrated how the use of an ultrasonic horn accelerates the removal of remnant support material layers from the Cleaning Challenge Model A. This is relevant for the cleaning of small features or fragile objects requiring refined post-processing. Optical quantification is a suitable method for measuring remaining support material on outer surfaces, although it cannot be used for quantification of cleaning inside channels printed in opaque materials.
The results for the Cleaning Challenge Models B.1 and B.2 show that channels of diameter $0.5-5 \mathrm{~mm}$ could be cleaned with an ultrasonic horn up to a certain depth that depends on the diameter of the channel, the ultrasound power setting, and the liquid used. This is confirmed by the fact that the removal rates for the first (non-contact) $20 \mathrm{~mm}$ and the final $20 \mathrm{~mm}$ (also non-contact) are similar. Streaming and/or cavitation lead to cleaning up to $2 \mathrm{~cm}$ in front of the tip. Large channels were cleaned better than small channels, especially when the ultrasonic horn tip could enter the channel, with a cleaning rate of $7.5 \mathrm{~mm} / \mathrm{min}$; the largest depth reached was $7 \mathrm{~cm}$. However, when the ultrasonic horn tip enters the channel, it is very likely to touch the walls and channel entrance. This leads to damage of both the printed model and the ultrasonic horn tip, and should be avoided. When the tip could not enter the channel (any further), then the cleaning rate was reduced by approximately 3 times, and the final depth was limited to $20 \mathrm{~mm}$ (large channel, $70 \%$ power setting) down to $5 \mathrm{~mm}$ (smallest channel, $50 \%$ power setting). This suggests that the cavitation bubbles and the streaming induced by the ultrasonic horn are capable of removing support material, but only in the vicinity of the ultrasonic horn tip; thinner and curved channels may have even less penetration depth. The speed of sound of the support material is unknown, but assuming it is similar to that of water $(1500 \mathrm{~m} / \mathrm{s})$, the wavelength at $30 \mathrm{kHz}$ would be on the order of a few $\mathrm{cm}$. While this suggests that standing waves fit inside the channel, it is likely that the support material dampens the ultrasound significantly (as with many soft materials). Therefore acoustic reflections are expected to play a minor role.

While ultrasonic baths have a much larger working area (or volume), the cavitation process inside baths is a random, unfocused process and consequently, the cleaning is much slower than with an ultrasonic horn. For practical situations, there is a trade-off 


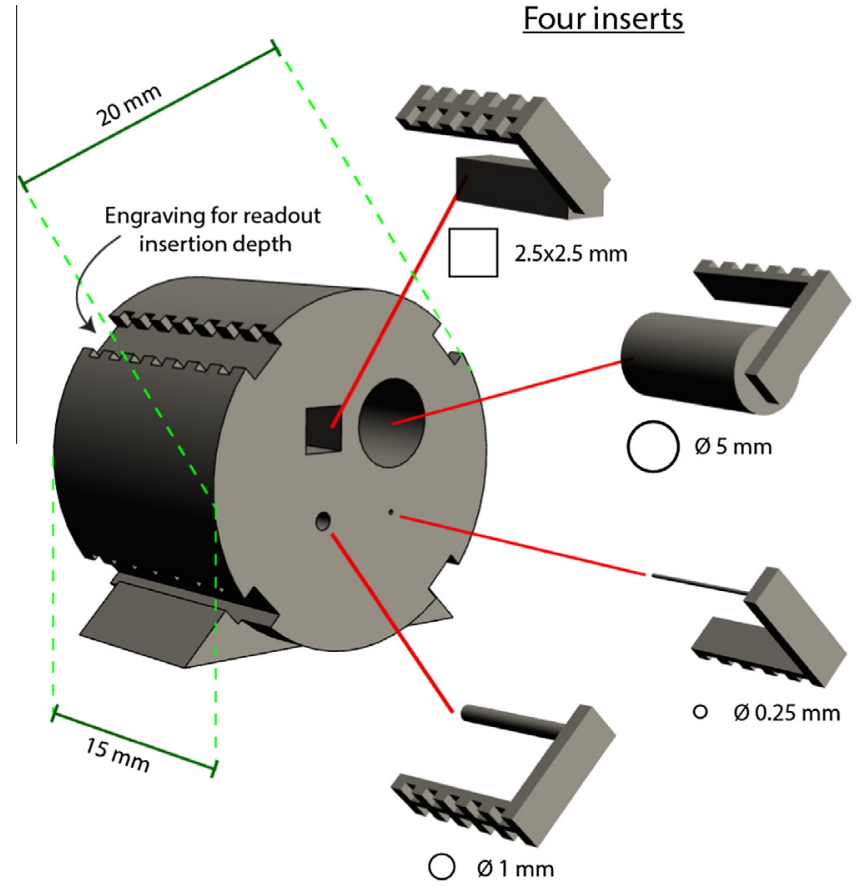

Fig. 14. Schematics of the CCD benchmark model with inserts rods. The corresponding shaped rods are inserted in the holes and a scale can be used to measure the penetration depth. The quality of the cleaning protocol can be assessed in a simple way.

between fast, local cleaning with an ultrasonic horn, and slower cleaning of entire objects with an ultrasonic bath. A combination, in which an ultrasonic horn is used to clean the remaining small details not removed by an ultrasonic bath may also be useful. Commercially available equipment for contact-less cleaning can also be used as an alternative; for example, a nozzle generates a megasonic wave $(600 \mathrm{kHz})$ through a flowing liquid to a small area of ca. $4 \mathrm{~mm}$ on a substrate [31].

Based on our observations, the contribution of chemical dissolution by $\mathrm{NaOH}$ is minor when using the ultrasonic horn. Fig. 13b shows that most of the cleaning is done by mechanical forces, with only a small increase in removal rate or depth when $\mathrm{NaOH}$ is used instead of water. We consider that the time scale of chemical dissolution effects is slower than that of the mechanical removal forces. These results may not be statistically significant since there is a variation in the results from the manual operation of the ultrasonic horn, which is difficult to account for.

\subsection{Cleaning Challenge Device (CCD) design proposition}

As discussed in the introduction, the evaluation of "what is clean" needs to be further investigated. The answer is application dependent, since e.g. mechanical models have less stringent cleanliness requirements than medical implants. For each application a robust evaluation of the cleaning techniques is required. For this reason we have designed a special Cleaning Challenge Device (CCD) that we make freely available online [32] so that, in conjunction with the experimental results presented in this article, scientists and users of 3D printing tools can use and share their experiences.

Inspired by the Cleaning Challenge Models used in this study, our CCD (shown in Fig. 14) was designed to include, in a simple geometrical object, the most important and challenging features to be cleaned: small close-end holes with different cross sections, ridges, etc. The model consists of a cylindrical body $(20 \mathrm{~mm}$ in radius, $15 \mathrm{~mm}$ in length), with a total volume of $4.43 \mathrm{~cm}^{3}$. The diameter of each hole in the body was chosen to represent different resolutions of the most common printers currently on the market; the $0.25 \mathrm{~mm}$ diameter rod and circle correspond to only a few layers in high-resolution printers. The depth of insertion of a rod in a corresponding hole printed in the main body of the model can be used to evaluate if the support material was removed inside. Additionally, the friction due to the rotation of a (cylindrical) rod inside the hole gives a secondary indication of the quality of the cleaning.

We have used this CCD to test different ultrasonic cleaning protocols in our lab. This has reduced our typical cleaning procedures for 3D printed objects in an ultrasonic bath from $8 \mathrm{~min}$ to $1 \mathrm{~min}$. We encourage other researchers or 3D printing enthusiasts to incorporate such protocol and CCD in their post-processing routines. We aim at making this CCD a benchmark model for investigating the capabilities of cleaning techniques, but also to compare different cleaning procedures. It can serve as a periodic quality control tool as well, since it can evidence whether the cleaning procedures and devices still function properly with the passing of time.

\section{Conclusions}

The use of ultrasonic cavitation has been demonstrated to have a positive effect in the removal of support material with relevance for the 3D printing objects, specifically Objet Eden 350V. The use of sodium hydroxide $(\mathrm{NaOH})$ solutions showed also an improved removal rate at the two concentrations studied $(0.5$ and $6 \mathrm{M})$. The optimal conditions found for the removal were those using $6 \mathrm{M} \mathrm{NaOH}$ in combination with ultrasound, but for some applications the $0.5 \mathrm{M}$ offers milder basic conditions with good cleaning effect. Additionally, the liquid could be warmed up to $\sim 30^{\circ} \mathrm{C}$ in order to enhance the chemical dissolution. It remains to be seen, e.g. with SEM or other analysis methods, if the $\mathrm{NaOH}$ solutions have no effect on the printed material surface properties after the cleaning procedure.

We introduce and make available a Cleaning Challenge Device (CCD) that can help in the assessing of post-processing of 3D printed materials. This device can also be used in the monitoring of other processes, or characterization of equipment effectiveness in the removal of different contaminants.

\section{Acknowledgements}

We thank Kleizen Modelmakerij BV for printing the test models used in this study, and for valuable discussions. We acknowledge NanoLabNL for the voucher that allowed us to make SEM images. We thank Mark Smithers for assistance with the SEM microscope. Finally, we acknowledge Prof. Gardeniers and Prof. M. Ashokkumar for fruitful discussions.

\section{References}

[1] Ge Q Qi HJ, Dunn ML. Active materials by four-dimension printing. Appl Phys Lett 2013;103:131901.

[2] Symes MD et al. Integrated 3D-printed reactionware for chemical synthesis and analysis. Nat Chem 2012;4:349-54.

[3] Dunn JJ, Hutchison DN, Kemmer AM, Ellsworth AZ, Snyder M, White WB et al 3D printing in space: enabling new markets and accelerating the growth of orbital infrastructure. In: Proc. Space Manufacturing 14: Critical Technologies for Space Settlement. Space Studies Institute, 29-31 October 2010, Mountain View, CA.

[4] Moroni L, Nandakumar A, de Groot FB, van Blitterswijk CA, Habibovic P. Plug and play: combining materials and technologies to improve bone regenerative strategies. J Tissue Eng Regen Med 2013. http://dx.doi.org/10.1002/term.176. n/a-n/a.

[5] Moroni L, de Wijn JR, van Blitterswijk CA. Integrating novel technologies to fabricate smart scaffolds. J Biomater Sci Polym Ed 2008;19:543-72. 
[6] Masood SH. Comprehensive materials processing. In: Advances in additive manufacturing and tooling, vol. 10; 2014. p. 1-2.

[7] Stampfl J, Liska R. New materials for rapid prototyping applications. Macromol Chem Phys 2005;206:1253-6.

[8] Walker WF. Ultrasonics in production processes. Ultrasonics 1963;1: 123-9.

[9] Crawford AE. The measurement of cavitation. Ultrasonics 1964;2:120-3.

[10] Niemczewski B. Observations of water cavitation intensity under practical ultrasonic cleaning conditions. Ultrason Sonochem 2007;14:13-8.

[11] Bhirud U. Ultrasonic bath with longitudinal vibrations: a novel configuration for efficient wastewater treatment. Ultrason Sonochem 2004;11:143-7.

[12] Sališová M, Toma Š, Mason TJ. Comparison of conventional and ultrasonically assisted extractions of pharmaceutically active compounds from Salvia officinalis. Ultrason Sonochem 1997;4:131-4.

[13] Jenderka $K-V$, Koch $C$. Investigation of spatial distribution of sound field parameters in ultrasound cleaning baths under the influence of cavitation. Ultrasonics 2006;44:e401-6.

[14] Dular M, Osterman A. Pit clustering in cavitation erosion. Wear 2008;265:811-20.

[15] Birkin PR, Offin DG, Vian CJB, Leighton TG. Multiple observations of cavitation cluster dynamics close to an ultrasonic horn tip. J Acoust Soc Am 2011;130: 3379.

[16] Hauptmann M, Struyf H, De Gendt S, Glorieux C, Brems S. Importance of bubble size control in ultrasonic surface cleaning by pulsed high-frequency sound fields. ECS J Solid State Sci Technol 2013;3:N3032-40.

[17] Bram Verhaagen, David Fernández Rivas. Measuring cavitation and its cleaning effect. Ultrason Sonochem, Available online 20 March 2015, ISSN 1350-4177. http://dx.doi.org/10.1016/j.ultsonch.2015.03.009.

[18] stratasys.com. <http://www.stratasys.com>.

[19] Nesladek P, Osborne S, Rode T. Comparison of cleaning processes with respect to cleaning efficiency. In: Behringer UFW, editor. 27th European mask and lithography conference, SPIE, vol. 7985; 2011. p. 79850P-79850P-10.
[20] Jueschke M, Koch C, Dreyer T. An erosion sensor based on a quartz crystal microbalance for quantitative determination of the cleaning efficiency in an ultrasonic vessel. Ultrason Sonochem 2014;21:1900-6.

[21] Offin D, Birkin P, Leighton T. An electrochemical and high-speed imaging study of micropore decontamination by acoustic bubble entrapment. Phys Chem Chem Phys 2014.

[22] Steam sterilization - gke - cleaning and sterilization monitoring. gke.de <http://www.gke.de/en/steam-sterilization.html>.

[23] BuBble bags aanvragen $\leftarrow$ BuBclean. bubclean.nl. <http://www.bubclean.nl/ bubble-bags-aanvragen/>.

[24] Bonding and Gluing | Stratasys. stratasys.com. <http://www.stratasys.com/solutionsapplications/finishing-processes/bonding-and-gluing>.

[25] Safety Data Sheet Objet Verowhiteplus RGD835. stratasys.com. <http://www. stratasys.com/ /media/Main/Secure/MSDS/Rigid\%200paque\%20Materials/ DOC-06124-Objet-VeroWhitePlus-RGD835-US.pdf>.

[26] Hauptmann M et al. Enhancement of cavitation activity and particle removal with pulsed high frequency ultrasound and supersaturation. Ultrason Sonochem 2013;20:69-76.

[27] Brown WH, Poon T. Introduction to organic chemistry. 5th ed. Wiley Global Education; 2012.

[28] Riesz P, Kondo T. Free radical formation induced by ultrasound and its biological implications. Free Radical Biol Med 1992;13:247-70.

[29] Riesz P, Berdahl D, Christman CL. Free-radical generation by ultrasound in aqueous and nonaqueous solutions. Environ Health Perspect 1985;64: 233-52.

[30] Makino K, Mossoba MM, Riesz P. Chemical effects of ultrasound on aqueoussolutions - formation of hydroxyl radicals and hydrogen-atoms. J Phys Chem 1983;87:1369-77.

[31] Megasonic single-/dual-nozzle-Sonosys GmbH. sonosys.de. <http:// www.sonosys.de/products/single-dual-nozzle/view?set_language=en>.

[32] Cleaning Challenge Device for 3D printing $\leftarrow$ BuBclean. bubclean.nl. <http:// www.bubclean.nl/cleaning-challenge-device-for-3d-printing/>. 